\title{
Giant cystic lymphangioma originating from the cardia of the stomach: A case report
}

\author{
GENG CHEN, MINGQING LIU, TAYYAB HAMID MALIK, SHOUYING LI, YING TANG and HONG XU \\ Department of Gastroenterology, The First Hospital of Jilin University, Changchun, Jilin 130021, P.R. China
}

Received December 24, 2014; Accepted January 11, 2016

DOI: $10.3892 /$ etm.2016.3090

\begin{abstract}
Cystic lymphangiomas are rare benign tumors involving the neck, head, and axilla, and most frequently occur in children before the age of 5 years. In the present study, the case of a giant cystic lymphangioma originating in the cardia of the stomach was reported in an 18-year-old female complaining of abdominal distention. Contrast-enhanced computed tomography and endoscopic ultrasonography revealed a large, multilobulated cystic mass located between the cardia and esophagogastric junction with a diameter of $4.0 \mathrm{~cm}$. The lesion was successfully removed by endoscopic submucosal dissection. Subsequent immunohistochemical analysis of the lymphatic endothelium-specific O-linked sialoglycoprotein D2-40 confirmed the diagnosis of cystic lymphangioma. No complications associated with the tumor dissection occurred, and the patient did not report any further complaints or any signs of recurrence at 6- and 18-month follow-up. The present case demonstrates that a diagnosis of cystic lymphangioma should be considered in non-pediatric patients suffering aspecific abdominal complaints.
\end{abstract}

\section{Introduction}

Lymphangiomas are rare malformations of the lymphatic system that present as benign submucosal tumors, and largely dilated lymph channels and cisterns (1). These structures typically occur during early childhood $(90 \%$ occur in patients $<2$-years-old) and account for $\sim 4 \%$ of all vascular tumors in children. These tumors are usually located in the head, neck and axilla (2). Nevertheless, lymphangiomas may occur at any age and involve any part of the body; thus, a diagnosis of lymphangioma should always be considered when investigating an unexplained mass.

Correspondence to: Dr Hong Xu, Department of Gastroenterology, The First Hospital of Jilin University, 71 Xinmin Street, Changchun, Jilin 130021, P.R. China

E-mail: chxuhong@163.com

Key words: cystic lymphangioma, endoscopic submucosal dissection, cardia
Although lymphangiomas of the gastrointestinal tract are very rare, studies have previously reported such tumors originating from the esophagus and stomach $(3,4-7)$. The majority of cystic lymphangioma cases are asymptomatic and, as such, misdiagnosis rates remain high (8). Since lymphangioma are benign, treatment is not usually required unless the legion is particularly large. Common treatments include laparoscopy and surgical resection (5).

The present study adds to the current literature by reporting the case of 18-year-old female with cystic lymphangioma arising from the cardia of the stomach. The patient presented with abdominal distention and diagnosis of lymphangioma was confirmed by histopathological analysis. The lesion was successfully treated with endoscopic submucosal dissection (ESD). Following ESD, the patient recovered quickly and fully, and no further symptoms or complaints were reported. This distinctive case demonstrated that a diagnosis of gastrointestinal lymphangioma should always be considered at any age, and that ESD is an acceptable definitive treatment strategy for such cases.

\section{Case report}

In September 2013, an 18-year-old female presented at The First Hospital of Jilin University (Jilin, China) with unexplained abdominal distention lasting 3 months. The patient had no other gastrointestinal symptoms, such as stomachache, nausea or emesis. Routine clinical tests, including a physical examination and standard laboratory tests, were unremarkable and thus, an endoscopic examination was required. The patient underwent gastroscopy (Olympus GIF-Q260J; Olympus Corporation, Tokyo, Japan) in September 2013 at the Department of Gastroenterology at The First Hospital of Jilin University, which revealed a large submucosal mass between the cardia and esophagogastric junction, measuring $3.0 \times 4.0 \mathrm{~cm}$. The mass was soft and easily deformed by pressure, and it had a smooth surface without ulceration or erosion (Fig. 1A). The possibility of submucosal lesions indicated further investigation using computed tomography (CT), which revealed the presence of a low density nodular mass with a diameter of $\sim 4.0 \mathrm{~cm}$ protruding into the gastric cavity and located anteromedial to the cardia, proximal to the esophagogastric junction, and adjacent to the left lobe of the liver (Fig. 1B). The aspect of the mass was slightly enhanced when investigated using contrast-enhanced CT (Ultravist 300; 

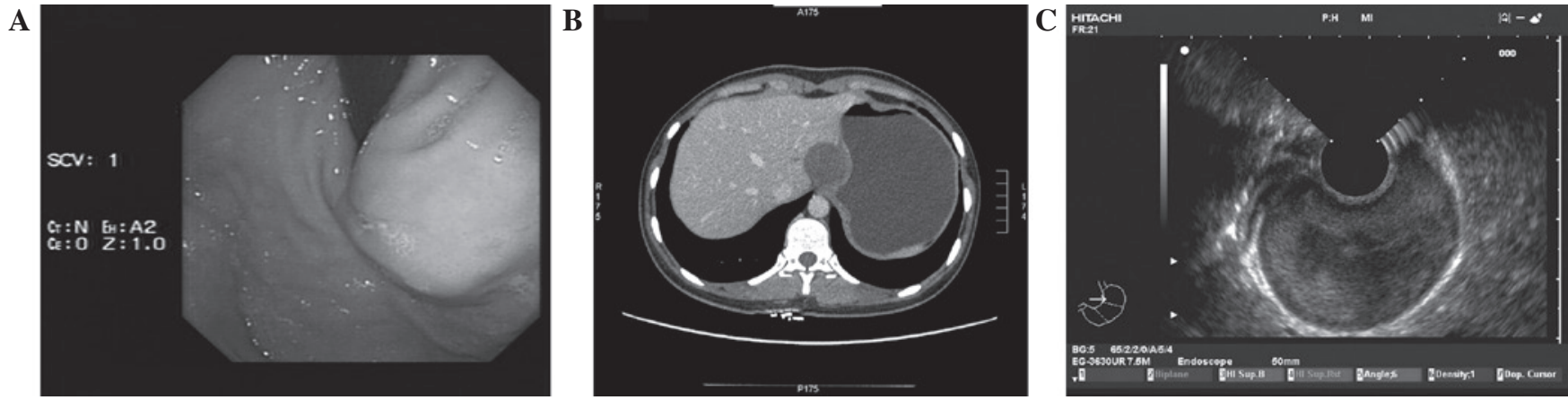

Figure 1. Diagnosis of gastric cystic lymphangioma. (A) Endoscopic view of a bulge of the gastric mucosa of the cardia. (B) Abdominal computed tomography scan revealing a low density nodular mass protruding into the gastric cavity, located anteromedial to the cardia and the last portion of the esophagus, and adjacent to the left lobe of the liver. (C) Endoscopic ultrasound view of a homogenous medium high echo, originating from the submucosal layer.

A

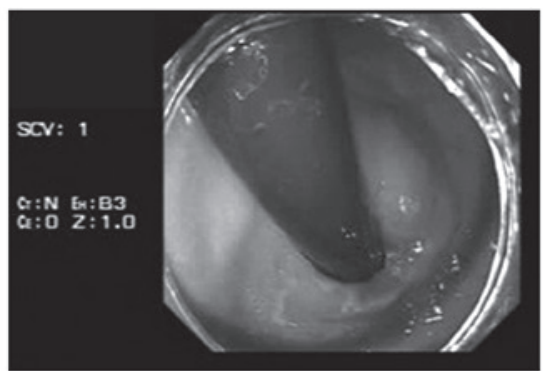

D

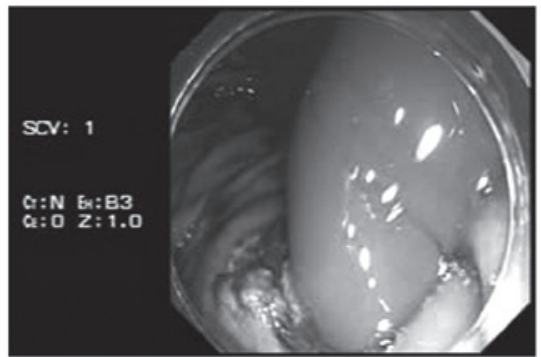

B

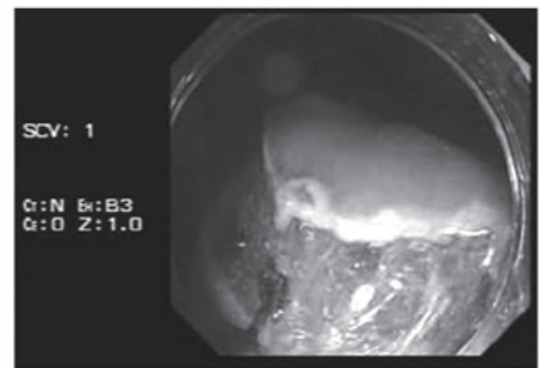

$\mathbf{E}$

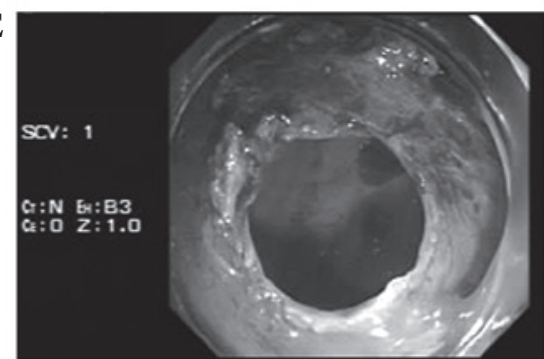

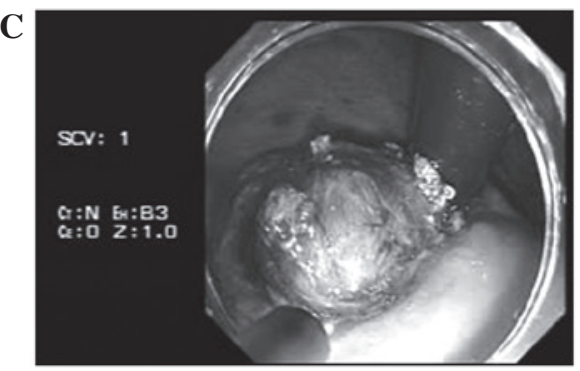

F

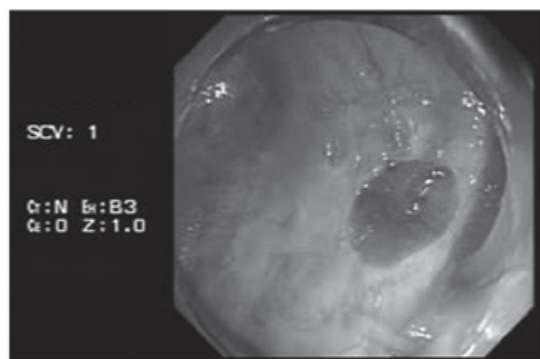

Figure 2. Resection procedure. (A) Endoscopic view of a bulge of the gastric mucosa of the cardia; (B) dissected lesion along submucosa; (C) first view following mucosa incision; (D) status after opening of the lesion (a brown viscous liquid was found to be flowing from within the lesion); (E) status after endoscopic submucosal dissection of the partial cystic wall (a cystic cavity was observed); and (F) well-demarcated hyperemic spots observed on the inner wall.

Bayer Corporation, Leverkusen, Germany). Endoscopic ultrasonography (EUS) using a UM-DP 20-25R probe (Olympus Corporation) was performed to further examine the nature of the mass. This procedure resulted in the detection of a homogenous and medium high-echo lesion, measuring $3.4 \times 2.4 \mathrm{~cm}$ and located in the submucosal layer (Fig. 1C). The lesion was not clearly separated from the muscle layer and did not display evidence of invasion or a blood vessel component. As it was suspected that the lesion was of a cystic nature, further EUS-guided fine-needle aspiration biopsy collection was not attempted.

Based on the aforementioned results, ESD with intravenous $20 \mathrm{ml}$ propofol (0.2 g; Fresenius Kabi AB Corporation, Beijing, China) anesthesia was selected as the therapeutic intervention for the preoperative diagnosis of a cystic lesion. First, visualization of the lesion was improved by retroflexion of the fundus of the stomach (Fig. 2A). The lesion was then elevated by submucosal injection of $0.4 \%$ sodium hyaluronate (Jingfeng Corporation, Shanghai, China). Subsequently, the mucosal surface was dissected, exposing a submucosal lesion that was soft and exhibited a white fiber membrane surrounding its surface (Fig. 2B and C). An electric knife (KD640-L; Olympus Corporation) was used to open the lesion, causing release of ample brown viscous fluid flowing from the core of the lesion (Fig. 2D). Subsequent to washing with $0.9 \%$ saline, a smooth wall with well-demarcated hyperemic spots was observed (Fig. 2E and F). The procedure ended with the cystic lumen remaining open, without closure of the dissected site. The partial resection of the cystic wall and hyperemic mucosa were examined by histopathology, including immunohistochemistry for the lymphatic endothelium-specific 40-kDa O-linked sialoglycoprotein D240 using a two-step Max-Vision kit, according to the manufacturer's protocol (Zhongshan Jinqiao Biotechnology Co., Ltd., Beijing, China). The results revealed the presence of highly-dilated lymphatic channels and D2-40-positive lymph vessels (Fig. 3A and B), which were consistent with a diagnosis of submucosal lymphangioma. The patient was discharged without any complications after 5 days and tolerated a regular diet. At 1 week after the surgery, gastroscopy demonstrated ulceration at the site of ESD, which was in line with the normal postoperative changes expected following the procedure (Fig. 4A). At 6-month follow-up, 

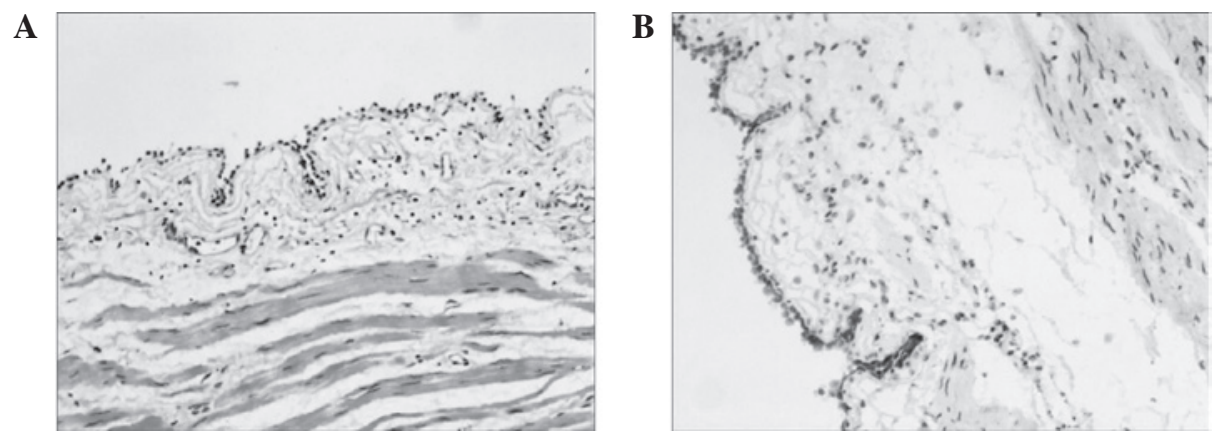

Figure 3. Histopathological diagnosis of gastric cystic lymphangioma. (A) Microscopic view of the specimen, depicting various lymphatic spaces (hematoxylin-eosin staining; magnification, x400); and (B) endothelial lining of cysts (D2-40 immunostaining; magnification, x400).
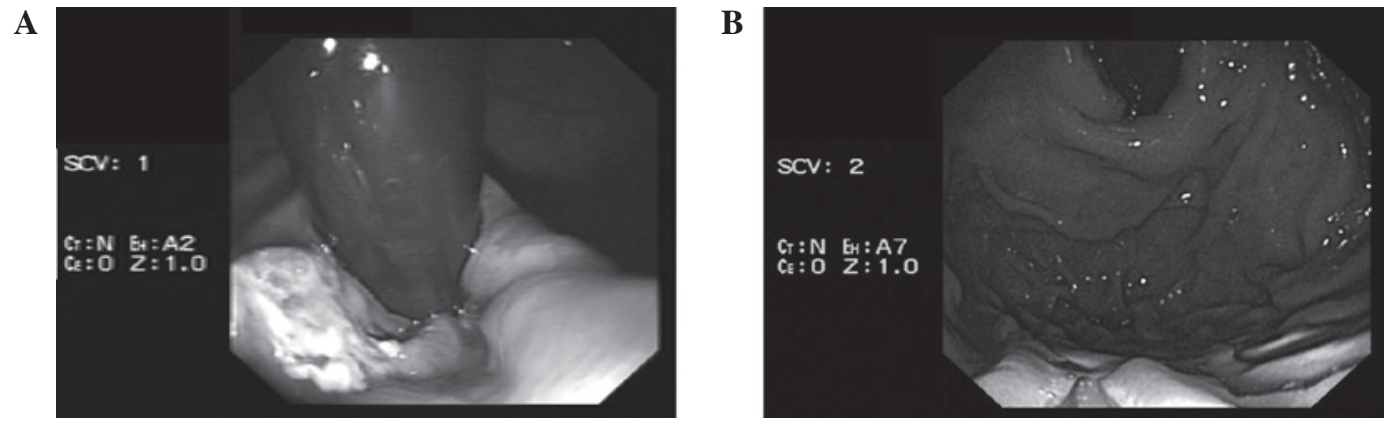

Figure 4. Follow-up gastroscopy examination. (A) At 1 week after the surgery, gastroscopy revealed an ulcer on the site of the endoscopic submucosal dissection. (B) After 6 months, gastroscopy showed a normal mucosa without signs of recurrence.

gastroscopy demonstrated that the ulcer had healed and the mucosa of the cardia was smooth without any signs of recurrence (Fig. 4B). The patient reported no further symptoms or discomfort. The last follow-up was in March 2015; gastroscopy demonstrated there was no recurrence and the patient was not suffering from any discomfort. Written informed consent was obtained from the patient prior to publication.

\section{Discussion}

Endoscopy and EUS have become indispensable tools for diagnosing and differentiating gastric submucosal lesions and other gastric tumors (9). With the increasing number of patients undergoing endoscopic evaluation, it is to be expected that largely asymptomatic cases of gastrointestinal system-associated masses will be detected more frequently (10). In the present study, a case of a giant cystic lymphangioma arising from the cardia was reported in an 18-year-old patient complaining of abdominal distention. The lesion was treated by ESD, and the results of the pathological examination were consistent with a diagnosis of cystic lymphangioma.

Lymphangioma originating from the gastrointestinal tract is very rare. The condition typically occurs in the head, neck and axilla, and the vast majority of cases occur in young children (2). Its detection in adolescents has been rarely reported; however, as the present study demonstrates, with increased endoscopic surveillance the detection of such cases may increase $(3-5,10)$. Thus, gastroenterologists should be aware of the possibility of this type of lesion. Although the etiology of cystic lymphangiomas is not fully understood (11), anomalous development of the lymphatics or inflammation and obstruction of developed lymphatic channels have been proposed as possible mechanisms (3). To date, only a small number of studies have described cystic lymphangiomas occurring intra-abdominally in adolescent patients (12). In particular, only four cystic lymphangiomas originating from the lesser curvature of the stomach have been documented $(3-5,10,13)$. The present study described cystic lymphangioma arising from the cardia and the anterior wall of the distal body. Although the majority of cases occur in young children, clinical presentation can be highly diverse and may include chronic abdominal pain, hunger pain, sour regurgitation, nausea and weight loss (5). Clinically, the majority of cases are asymptomatic and thus detected incidentally. In the current case, the patient presented with a 3-month history of abdominal distension, and the diagnosis of lymphangioma can be considered to be coincidental based on its non-specific clinical presentation. Therefore, it can be predicted that a large number of cases of lymphangioma remain undetected.

With respect to the diagnosis of cystic lymphangioma, CT constitutes an important imaging modality in which the aberration presents as a non-enhancing extramucosal mass with homogeneous attenuation, allowing evaluation of the extent of the lesion before the treatment commences (14). In the case of gastric cystic lymphangioma, the disease can manifest itself as a submucosal polypoid mass with an endoscopically smooth surface. Upon physical manipulation by forceps, the mass appears softer than its surroundings. Furthermore, a cystic echo pattern during EUS characterizes gastric cystic lymphangiomas $(6,7)$. Differential diagnoses for submucosal tumors of the stomach include lipoma, hemangioma, mesenchymoma, leiomyoma and other tumors (15). Clinically and radiologically, it is difficult for these lesions to be distinguished from each other. Endoscopy 
and EUS are important diagnostic tools for this purpose, as they can establish whether the tumor is located in the mucosa or submucosa, thus aiding the selection of operative procedures, such as endoscopic resection or surgery (16). Regarding histopathology, D2-40 is a specific marker of lymphatic endothelial cells, therefore if positive expression is detected in the lymph vessels then reaching a diagnosis of cystic lymphangioma is relatively straightforward. Cyst contents are variable and may include serous, hemorrhagic, chylous or mixed fluids (17).

Lymphangiomas are benign and do not typically require medical treatment, although patients may select surgical removal for personal reasons or to prevent local invasion. Treatment options include endoscopic removal of lesions $<2 \mathrm{~cm}$ or surgical resection of larger lesions (18). The main choice of treatment is surgical resection, usually performed via laparoscopy, which has good safety and efficacy. Generally, surgical resection is only applied to larger lesions and endoscopy appears to be a viable alternative. Endoscopy and EUS can be used to determine the size and precise location of the lesion. Endoscopic mucosal resection and ESD are the most widely used endoscopic resection methods (6). They are considered to be minimally invasive and safe, while complication rates are less than those of surgery. Endoscopic minimally invasive treatment has evident advantages over traditional treatments and was successfully applied in the present case. Nevertheless, open surgery remains an option for the management of lymphangioma for lesions that are large or not suitable for endoscopy.

In conclusion, the present study reported an unusual case of histologically confirmed cystic lymphangioma arising from the cardia of the stomach, which was studied by gastroscopy, EUS and CT, and treated by ESD. A review of the literature supports that endoscopic treatment of lymphangioma, in relation to its size, location and the absence of complications, is an appropriate method. In addition, the present case demonstrated that lymphangioma should be considered when adolescent patients present atypical symptoms.

\section{References}

1. Okazaki T, Iwatani S, Yanai T, Kobayashi H, Kato Y, Marusasa T, Lane GJ and Yamataka A: Treatment of lymphangioma in children: Our experience of 128 cases. J Pediatr Surg 42: 386-389, 2007.

2. Grasso DL, Pelizzo G, Zocconi E and Schleef J: Lymphangiomas of the head and neck in children. Acta Otorhinolaryngol Ital 28: $17-20,2008$.
3. Leland HA, Lee JT, Tan JH, Romine LE and Bansal V: Cystic lymphangioma of the lesser curvature of the stomach-case report. J Radiol Case Rep 5: 31-37, 2011.

4. Gockel I, Muller H, Kilic M, Eitelbach F, Gaedertz Chr and Peters H: Giant cystic lymphangioma of the stomach. Eur J Surg 167: 927-930, 2001.

5. van Oudheusden TR, Nienhuijs SW, Demeyere TB, Luyer MD and de Hingh IH: Giant cystic lymphangioma originating from the lesser curvature of the stomach. World J Gastrointest Surg 5: 264-267, 2013.

6. Zhao ZF, Kuang L, Zhang N, Ma SR, Yang Z, Han X, Zhao YF, Gao F, Gong ZJ and Yang L: Endoscopic diagnosis and treatment of esophageal cavernous lymphangioma. Surg Laparosc Endosc Percutan Tech 23: 299-302, 2013.

7. Arashiro M, Satoh K, Osawa H, Yoshizawa M, Nakano H Ajibe H, Miura Y, Yoshida T, Hirasawa T, Yamamoto H and Sugano K: Endoscopic submucosal dissection of esophageal lymphangioma: A case report with a review of the literature. Clin J Gastroenterol 3: 140-143, 2010.

8. Hoffmann J, Kirschniak A, Scharf G, Feilitzsch VM, Konigsrainer A and Zdichavsky M: Laparoscopic resection of a lymphangiomatous cyst of the colon: A case report. J Med Case Rep 5: 431-434, 2011.

9. Kang JH, Lim JS, Kim JH, Hyung WJ, Chung YE, Choi JY, Park MS, Kim MJ and Kim KW: Role of EUS and MDCT in the diagnosis of gastric submucosal tumors according to the revised pathologic concept of gastrointestinal stromal tumors. Eur 19: 924-934, 2009.

10. Kim HS, Lee SY, Lee YD, Kim DH, Kwon JG, Tak WY, Kweon YO, Kim SK, Choi YH and Chung JM: Gastric lymphangioma. J Korean Med Sci 16: 229-232, 2001.

11. Weeda VB, Booij KA and Aronson DC: Mesenteric cystic lymphangioma: A congenital and an acquired anomaly? Two cases and a review of the literature. J Pediatr Surg 43: 1206-1208, 2008.

12. Roisman I, Manny J, Fields S and Shiloni E: Intra-abdominal lymphangioma. Br J Surg 76: 485-489, 1989.

13. Alvite Canosa M, Alonso Fernandez L, Seoane Vigo M, Pérez Grobas J, Berdeal Díaz M, Bouzón Alejandro A, Carral Freire M, Budiño Ramos J and Gómez Freijoso C: Abdominal cystic lymphangioma in a teenager. Rev Esp Enferm Dig 100: 517-518, 2008.

14. Zhu H, Wu ZY, Lin XZ, Shi B, Upadhyaya M and Chen K: Gastrointestinal tract lymphangiomas: findings at $\mathrm{CT}$ and endoscopic imaging with histopathologic correlation. Abdom Imaging 33: 662-668, 2008.

15. Papanikolaou IS, Triantafyllou K, Kourikou A and Rösch T: Endoscopic ultrasonography for gastric submucosal lesions. World J Gastrointest Endosc 3: 86, 2011.

16. Hizawa K, Matsumoto T, Kouzuki T, Suekane H, Esaki M and Fujishima M: Cystic submucosal tumors in the gastrointestinal tract: endosonographic findings and endoscopic removal. Endoscopy 32: 712-714, 2000.

17. Levy AD, Cantisani V and Miettinen M: Abdominal lymphangiomas: Imaging features with pathologic correlation. AJR Am J Roentgenol 182: 1485-1491, 2004.

18. Irisawa A and Bhutani MS: Cystic lymphangioma of the colon: Endosonographic diagnosis with through-the-scope catheter miniprobe and determination of further management. Dis Colon Rectum 44: 1040-1042, 2001. 